\title{
The impact of patient information leaflets
}

\author{
The immediate effect on knowledge, attitudes and intentions in primary care attenders of a patient information \\ leaflet: a randomised control trial replication and extension
}

G. M. Humphris and E. A. Field Br Dent J 2003; 194: 683-688

\section{Aim}

To determine whether the influence of a leaflet on mouth cancer improves knowledge, related attitudes and intention to accept a mouth screen.

\section{Design}

Randomized controlled trial.

\section{Setting}

Dental and medical waiting rooms in the North West of England.

\section{Sample}

Nine hundred and forty nine patients from 16 practices were invited to participate.

\section{Measures}

Standardised multi-item scales of six outcome measures including knowledge, beliefs and intention to accept an oral cancer screen.

\section{Procedure}

A patient information leaflet was given to a randomised intervention group of patients. A single sheet questionnaire was completed by both groups of patients (immediately following leaflet administration in intervention arm of study).

\section{Statistical analysis}

$t$ tests were used to compare outcome variables between patients with and without access to the leaflet with Boneferroni correction.

\section{Results}

Participation rate was high $(91 \%)$. Knowledge $(p<0.001)$ and intentions $(p=0.003)$ benefited from patient access to leaflet. Anxiety was not raised with leaflet exposure. Some beliefs about the screening procedure appeared to be slightly improved by reading the leaflet $(\mathrm{p}<0.05)$.

\section{Conclusion}

This study supports previous findings of an immediate positive effect of an information leaflet on patients' knowledge of oral cancer and willingness to accept an oral cancer screen.

\section{IN BRIEF}

- A well-designed information leaflet has the potential to raise immediate knowledge levels of oral cancer in patients attending primary care facilities.

- Anxiety about an oral health screen appears not to be raised by such written material.

- Patients may be more willing to receive an oral health screen following access to the leaflet.

- Patient information leaflets are inoffensive for the patient, and inexpensive and time neutral for the practice.

\section{COMMENT}

Sheldon et $a l^{1}$ point out that 'health promotion practitioners often base their practice on opinion, received wisdom, or a favoured theory, occasionally supported by selective reference to a few studies of variable quality which rarely assess health outcomes' and they warn that health promotion activity needs to be evaluated rigorously.

A dentist seeking evidence for effectiveness of health promotion needs to decide what constitutes evidence as Springett ${ }^{2}$ suggests that, 'the most appropriate paradigm for evaluating health promotion is different from that currently being used within clinical medicine.'

You need to ask yourself:

- What evidence is there that the change has not been caused by other factors external to the health promotion?

- What population has been chosen?

- What changes are being looked for?

- What are the short and long term outcomes?

- Were measurements made before and after or were control groups used?

- Was blinding possible given that the intervention may have included a participatory role?

Speller et $a^{\beta}$ point out that evaluation should not just include outcome evaluation but also the implementing process - 'was it applied in the manner intended, did other factors come into play that might have affected the result, what did the participants think about the process?'

Humphris and Field's study is a well conducted randomized controlled trial using a previously developed method and based on knowledge of best practice. The outcomes were clearly stated and the study was well prepared with rigorous testing, an excellent response rate and details on non-responders. The largest impact is on increased knowledge and increased likelihood of accepting an oral screen. It is reassuring that the anxiety levels were not raised given this increased information.

How applicable are the results to your practice? Should you have these leaflets in your practice? The population was of a varied group of healthcare seekers. We do not know if the leaflets are useful in other ethnic groups who are non-English speaking which may be important in the context that oral cancer is more common in the Asian community. Humphries and Field are aware that immediate changes in knowledge and behaviour as a result of a one shot intervention is questionable when we know that attitudes and behaviours are shaped by a range of socio-economic and cultural influences and that a variety of methods may be needed over a longer period. ${ }^{3}$

Joanna M Zakrzewska, Senior Lecturer/ Hon Consultant in Oral Medicine, Barts and the London, Queen Mary's School of Medicine and Dentistry

1. Sheldon TA Sowden AJ, Lister-Sharp D. Search for evidence of effective health promotion. Letter, BrMed J 1998; 316: 703

2. Springett, J. Appropriate approaches to the evaluation of health promotion. Critical Public Health 2001; $11: 2$.

3. Speller $V$, Learmonth $A$, Harrison D. The search for evidence of effective health promotion BrMed J 1997; 315: 361-363. 